\title{
Messing up the membrane
}

The marine bacterium Vibrio parahaemolyticus induces cell death by injecting effectors into host cells through its type III secretion system 1 (T3SS1). The T3SS1-secreted effector VPA0450 contributes to host cell death, and Orth and colleagues now find that it achieves this by disrupting the interaction between the host cell membrane and the actin cortex.

Analysis of $V$. parahaemolyticus T3SS1 effector mutants revealed that those depleted of VPA0450

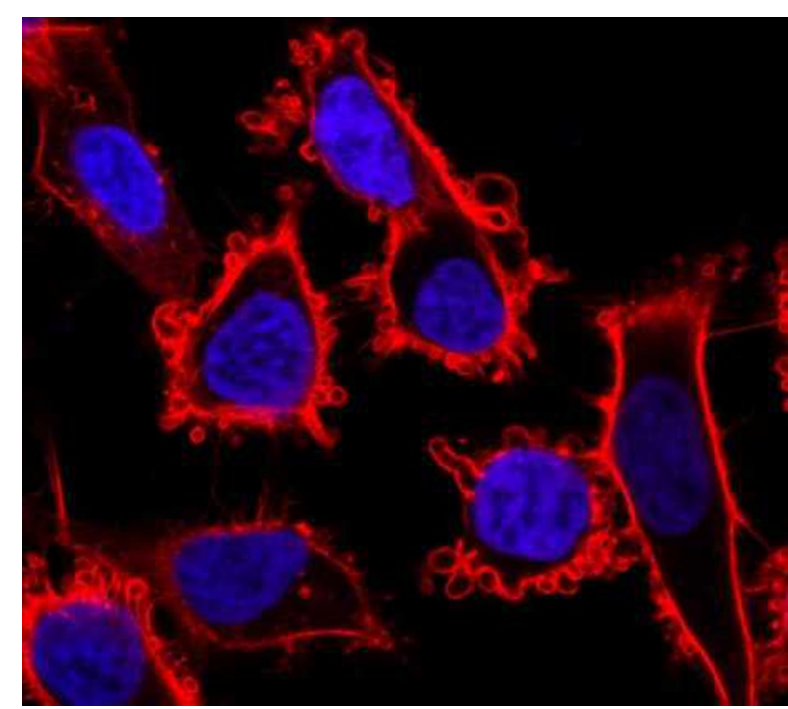

Membrane bleb formation in HeLa cells infected with Vibrio parahaemolyticus; actin (red) and the nucleus (blue) are shown. Image courtesy of C. A. Broberg, University of Texas Southwestern Medical Center, Dallas, USA. showed delayed host cell lysis and did not induce membrane blebbing. Furthermore, cells transfected with VPA0450 did not lyse but showed signs of membrane blebbing, indicating that VPA0450 interferes with the integrity of the host cell membrane.

VPA0450 contains five of the six catalytic motifs that are present in the active site of inositol polyphosphate 5-phosphatases (IPP5Cs), which dephosphorylate the D5 phosphate on inositol rings, so VPA0450 could act as an IPP5C. IPP5Cs have a structurally similar catalytic pocket to apurinic-apurimidinic endonucleases, which use a conserved His for function, and indeed cells transfected with a His356Ala mutant were morphologically similar to cells transfected with empty vector and showed reduced blebbing compared with cells transfected with wild-type VPA0450. Comparing the phosphatase activities of wild-type and His356Ala mutant VPA0450 confirmed that VPA0450 acts as an IPP5C, as wild-type, but not mutant, VPA0450 hydrolysed the D5 phosphate from phosphatidylinositol-4,5bisphosphate $\left(\operatorname{PtdIns}(4,5) \mathrm{P}_{2}\right)$ and PtdIns $(3,4,5) \mathrm{P}_{3}$.

PtdIns $(4,5) \mathrm{P}_{2}$ acts as a docking site for proteins that interact with the plasma membrane (such as phospholipase C $\delta 1$ (PLC $\delta 1)$ ), so hydrolysis of the D5 phosphate might lead to membrane blebbing by disrupting membrane docking. To examine this possibility, the authors transfected cells with a GFP-tagged pleckstrin homology (PH) domain from PLC $\delta 1$ (which tethers the actin cytoskeleton to the membrane). PH-GFP localized to the edge of the cells when co-transfected with the His356Ala mutant VPA0450, whereas it was found in the cytoplasm when cotransfected with wild-type VPA0450, confirming the authors' hypothesis.

Together, these findings show that the V. parahaemolyticus T3SS1-secreted effector VPA0450 contributes to the induction of host cell death by interfering with PtdIns signalling and preventing the interaction between the actin cytoskeleton and the plasma membrane. This leads to membrane blebbing, which the authors propose facilitates host cell lysis.

Rachel David

ORIGINAL RESEARCH PAPER Broberg, C. A. et al. A Vibrio effector protein is an inositol phosphatase and disrupts host cell membrane integrity. Science 19 Aug 2010 (doi:10.1126/ science.1192850) 